\title{
L'HOMME L'Homme
}

229 | 2019

Varia

\section{David Gibeault \& Stéphane Vibert, eds, Autorité et pouvoir en perspective comparative}

\author{
Aurélie Névot
}

\section{(2) OpenEdition}

1 Journals

Édition électronique

URL : https://journals.openedition.org/lhomme/33679

DOI : 10.4000//homme.33679

ISSN : 1953-8103

Éditeur

Éditions de l'EHESS

\section{Édition imprimée}

Date de publication : 1 mars 2019

Pagination : 185-188

ISSN : 0439-4216

\section{Référence électronique}

Aurélie Névot, « David Gibeault \& Stéphane Vibert, eds, Autorité et pouvoir en perspective comparative », L'Homme [En ligne], 229 | 2019, mis en ligne le 01 mars 2019, consulté le 08 janvier 2022. URL : http:// journals.openedition.org//homme/33679; DOI : https://doi.org/10.4000/lhomme.33679 


\section{HISTOIRE \& ÉPISTÉMOLOGIE}

David Gibeault \& Stéphane Vibert, eds Autorité et pouvoir en perspective comparative Paris, Presses de l'Inalco, 20I7, 388 p., bibl., index, fig. («TransaireS»).

$P$ graphique de Aliki Nono'ohimae Eerehau (1911-1984) donne le ton de cet ouvrage. «Dignitaire coutumier» de l'une des îles Salomon, il fut l'informateur privilégié de Daniel de Coppet (1933-2002), à qui ce volume collectif rend hommage. Mettre au premier plan visuel ce pourvoyeur de données ethnographiques fait sens, car les contributions ne portent pas sur les travaux de Daniel de Coppet mais proposent, sur la base de monographies très variées (Philippines, Papouasie-Nouvelle-Guinée, Chine, Polynésie, Tunisie, Russie du Xvi ${ }^{\mathrm{e}}$ siècle, Inde, Nouvelle-Calédonie), de poursuivre la discussion engagée par ce chercheur sur les concepts d'autorité et de pouvoir. Grâce au décentrement ethnographique de ces deux notions et donc à l'approche anthropologique - dont Daniel Gibeault souligne la spécificité (pp. 55-83) -, il s'agirait de "sortir de l'anthropologie potestative" pour promouvoir un certain «holisme structural» (Stéphane Vibert, pp. 11-53). Selon cette idée, l'ordre social ne saurait être réduit aux relations de pouvoir, mais devrait être appréhendé avec une approche holiste et relationnelle. C'est pourquoi le parti pris est ici, à la suite de Daniel de
Coppet et, donc, dans la lignée de Louis Dumont dont il s'est inspiré (évoqué par Stéphane Vibert, pp. 36-42), de revaloriser la notion d'autorité, non pas en la percevant comme un instrument du pouvoir, mais en la comprenant «à partir de son inscription dans l'espace symbolique d'une totalité sociale» (Stéphane Vibert, p. 17).

La discussion s'ouvre avec un article d'Élisabeth Luquin (pp. 87-111) sur le système d'autorité et d'efficacité rituelle chez les Mangyan Patag (Philippines). Dans leur système de pensée, ces derniers font prédominer la notion de 'āpu: «être en relation» ou «relation constitutive des êtres». Leurs ancêtres sont d'ailleurs non seulement appelés «les humains d'autrefois», mais aussi du nom même de «relation» (p. 87). L'ethnologue souligne que du rapport établi avec les ancêtres dépendent les autres relations établies dans la société mangyan, comme celle qui unit un corps et un esprit ou celles entre affins. Par le prisme de deux rituels complémentaires décrits avec force détails, elle prête plus spécifiquement attention au «fondement de l'autorité» de ces ancêtres, lequel conditionne «les pouvoirs" des officiants rituels. Élisabeth Luquin précise que l'idée de pouvoir est inappropriée 
à la culture locale, car le mot qui s'en approcherait renvoie davantage à l'efficacité, la potentialité, la force, l'influence, etc. On pourrait ajouter que la notion d'autorité ne semble pas non plus pertinente pour les représentations locales; c'est avant tout un maillage relationnel complexe, ponctué d'interdépendances structurelles, que nous permet de découvrir cet article.

Toujours en rapport à l'autorité abordée dans une perspective relationniste, mais cette fois-ci en Papouasie-Nouvelle-Guinée, Almut Schneider (pp. 115-134) se penche sur le rôle des "hommes éminents" (yie nuim), capables de parler et d'être à l'écoute d'autrui, lors des échanges cérémoniels appelés magadl. Ce ne sont toutefois pas leurs paroles conditionnant un retour à la normale qui prédominent, mais la raison (puglu) de la prestation donnée au cours de ces rituels et le nom du ou des donateur(s). L'ethnologue nous invite à suivre le déroulement d'un magadl organisé pour les enfants d'une suicidée, rite dont l'issue est le rétablissement des relations entre affins. Comme pour l'article précédent, les concepts de pouvoir et d'autorité ne semblent pas ici heuristiquement féconds. L'autrice note d'ailleurs, en conclusion de son article, que le concept d'autorité reste insatisfaisant pour caractériser ce qu'elle observe et que la relation prédomine (p. 131). On sent, une nouvelle fois, une forme de résistance du matériau ethnographique aux concepts.

David Gibeault propose ensuite d'introduire l'idée d' "autorisation" (pp. 137171). Selon lui, en Chine, "les pratiques associées aux autorisations irriguent la société tout entière» (p. 139); elles mettraient en jeu l'identité des personnes et des choses impliquées, et l'autorisation serait un acte de transmission, ce qui la distinguerait de "notre notion" de pouvoir. L'auteur part d'une glose (réductrice) de l'expression $x u$ yuan 许愿 que l'on traduit communément par "prière» et qu'il décompose en «autoriser» $(x u)$ et "rendre hommage» (yuan). Toute relation sociale chinoise serait fondée sur l'autorisation et l'hommage, lesquels formeraient une structure que l'on retrouverait dans les temples et dans d'autres champs de la société. L'auteur propose plus spécifiquement de s'intéresser aux relations d'autorisation des villageois de Wujiagou (province de Hubei, Chine centrale), en rapport à leurs temples et donc à leurs dieux. Un choix méthodologique est opéré "pour simplifier la présentation»: décrire au présent des visites au temple alors même que ces lieux ont disparu (p. 142). Une telle fiction narrative est d'autant moins convaincante que l'on rencontre ensuite un certain nombre d'approximations sinologiques que l'on ne pourra pas toutes relever ici (sur le dieu du Taishan et les déesses qui l'accompagnent, notamment). De même, lorsque David Gibeault évoque la «subordination" d'un dieu sur l'autre, il semble avant tout renvoyer à deux ancrages territoriaux différents, en référence à des temps de fondations distincts et à des spécialistes religieux probablement spécifiques.

Le quatrième chapitre (pp. 175-207) est au plus près de la problématique de l'ouvrage, avec une discussion critique des notions d'autorité et de pouvoir par l'étude subtile d'une société polynésienne. Sophie ChaveDartoen propose en effet d'analyser la notion de "chef» dans le royaume de Wallis à partir de quatre termes vernaculaires ('aliki, pule, tu'i, hau). Elle revient donc aux finesses des représentations locales, basées sur des relations socio-cosmiques complexes, accordant une grande importance aux ancêtres et à Dieu. Sa perspective analytique prend ainsi en considération les bouleversements sociaux vécus par les Wallisiens: les arrivées tongiennes, la christianisation (depuis 1837) et la modernisation. Grâce à une ethnographie fouillée, elle dresse un tableau diachronique des différents usages des termes locaux susmentionnés. Finalement, l'autrice montre que les Wallisiens n'emploient pas l'idée de pouvoir, mais distinguent deux ensembles sémantiques: l'un renvoie à l'usage de la force et de la contrainte physique et marque la limite de l'autorité du chef, l'autre se réfere à la protection, au rassemblement sous une autorité et suppose un rapport réciproque de protection entre le roi et le peuple.

C'est une perspective encore différente que nous permet de découvrir Véronique 
Pardo (pp. 213-242). Elle fait une lecture anthropologique de l'histoire régionale du Sud-Est tunisien pendant l'instauration du protectorat français. L'autrice retrace l'évolution du système politique local. Le protectorat a engendré un transfert du lieu de pouvoir, une extériorité complète de la gouvernance par rapport aux villages qui la «subissent"; cette extériorité ne pourrait dès lors pas être qualifiée de "source d'autorité". Véronique Pardo propose ensuite de se focaliser sur l'"autorité locale" du village de Douiret: les hommes mariés du village qui reçoivent et véhiculent la baraka (la force de leur[s] saint [s] musulman[s]).

C'est la Russie du Xvi ${ }^{\mathrm{e}}$ siècle qui est au cœur des développements de Stéphane Vibert (pp. 245-265), dont l'analyse renvoie à une approche explicitement maussienne et dumontienne. Son étude vise à comprendre sur quoi repose la légitimité de l'autorité du tsar dans son double rapport à la volonté divine et au consentement populaire. Il ouvre sa réflexion en rappelant certains faits historiques concernant la sacralité des tsars des $\mathrm{XVI}^{\mathrm{e}}$ et $\mathrm{XVII}^{\mathrm{e}}$ siècles au regard des souverains eux-mêmes (notamment Ivan le Terrible) et de leurs cours, pour ensuite s'intéresser au peuple pour lequel cette autorité est légitime: le règne est soumis à un choix divin et est identifié au sort du peuple qu'il doit mener au salut éternel. Il montre que le tsar oscille entre icône divine et figure de l'Antéchrist, et que ce phénomène de sacralisation de l'autorité du tsar entraîne la valorisation du peuple comme "théophore».

Le sixième chapitre nous ramène au contemporain, plus spécifiquement à l'Inde du Nord. Delphine Ortis centre son analyse sur un sanctuaire musulman fondé au $\mathrm{XIII}^{\mathrm{e}}$ siècle, où se jouent des conflits entre différents groupes sociaux qui s'en réferent à l'État et à ses cours de justice pour donner voix à leurs positions respectives. Les deux conflits sur lesquels elle s'arrête révèlent des tensions autour des échanges monétaires et des rites. Ils mettent en lumière les légitimités sur lesquelles s'appuient les gens du sanctuaire et l'autorité que de telles légitimités leur procurent: le sanctuaire et la vie qui s'organise autour sont soumis à l'autorité des desservants (attachés par des liens directs ou indirects à Ghāzī Miyān, le saint martyr qui a donné son nom au sanctuaire), du comité (conseil qui administre le sanctuaire) et de l'État (les membres de ces deux dernières institutions n'appartiennent pas à des familles particulières). Grâce à une description fine et claire en dépit de la complexité des différents systèmes d'autorité en jeu, l'ethnologue montre que trois systèmes de référence se compénètrent: l'islam, la hiérarchie des castes, et l'État, l'autorité des uns et des autres étant souvent disputée.

Dominik Bretteville se réfère plus encore que les autres contributeurs (hormis Stéphane Vibert) à Daniel de Coppet et à Louis Dumont. Elle ouvre un nouveau chapitre en s'intéressant à l'autorité de la monnaie cérémonielle et au pouvoir de l'argent chez les Paimboa de NouvelleCalédonie. Son propos porte sur les rapports entre le pouvoir économique de la monnaie et son «autorité» en tant que monnaie cérémonielle. Après avoir présenté les monnaies traditionnelles (les hegi, qui sont en fait des chapelets de perles de coquillages renvoyant à l'ancestralité) et le numéraire qui tend à le remplacer (lequel est également appelé hegi dans le cadre rituel, alors qu'il prend un autre nom lorsqu'il s'agit d'acheter des marchandises), l'autrice analyse la circulation des monnaies au cours des principales "cérémonies» paimboa. Elle met ainsi en évidence, au regard des «relations socio-cosmiques» et de «l'autorité» de cette monnaie, la permanence d'une hiérarchie de valeurs en dépit des changements historiques et des nouveaux objets qui intègrent la société. Ce qui est introduit par la colonisation est intégré dans leur système d'échange propre et catégorisé selon les logiques de pensée locales.

La Chine est de nouveau au premier plan dans le dernier chapitre avec une étude de Catherine Capdeville-Zeng sur les relations sociales établies au sein de groupes rock chinois. Ce travail est directement inspiré des travaux de Louis Dumont et de Daniel de Coppet (p. 332). S'appuyant sur une observation menée au début des années 1990 , 
l'autrice en distingue deux types qu'elle nomme, pour celui aux caractéristiques communautaires: le "groupe holiste» (en reprenant une expression employée par les rockers eux-mêmes pour désigner une forme idéale de groupe de rock chinois, p. 330) et, pour celui aux caractéristiques plus individualistes, centré autour d'un chef: le "groupe impérial». Partant de l'idée qu'en dépit des transformations sociales et des bouleversements historiques, des structures de pensée se perpétuent, Catherine Capdeville-Zeng vise à montrer que les rapports entre membres renvoient à des idéologies chinoises anciennes sur le pouvoir et l'autorité. Rappelant l'influence non seulement du confucianisme, du taoïsme, mais également de la pensée légiste jusqu'à aujourd'hui, elle souligne la nécessité de prendre cette dernière en considération pour saisir ce que nous donne à voir la Chine actuelle. Ainsi, le "groupe holiste", qu'elle aborde par le prisme d'un groupe rock de femmes (pp. 330-337), renverrait à la tradition confucéenne, tandis que le "groupe impérial», qu’elle analyse avec la figure emblématique du rockeur Cui Jian (pp. 337-346), serait proche de la tradition légiste.

Le parti pris de cet ouvrage, ouvertement d'obédience maussienne et dumontienne, ne donne pas véritablement la part belle à l'ethnographie, qui semblerait exprimer autre chose (comme le montrent certains articles plus ou moins explicitement). Ces remarques ne sauraient méconsidérer la richesse ethnographique de ces études, bien au contraire: les termes vernaculaires introduits renvoient à des représentations du monde que l'on regrette de ne pas voir toujours mises au premier plan de l'analyse dans ces approches relationnelles du social. Dans d'autres cas, lorsqu'elles le sont, elles paraissent parfois inscrites dans une logique conceptuelle exogène et forcée. Or, on s'attendrait à lire ce que l'ethnographie donne à signifier par elle-même conceptuellement.

Aurélie Névot 